\title{
Vierges guerrières et mères courage : le panthéon des communistes espagnoles en exil
}

Virgin Warriors and Brave Mothers: the Pantheon of Communist Women in Exile

\section{Mercedes Yusta}

\section{OpenEdition}

1 Journals

Édition électronique

URL : http://journals.openedition.org/clio/9403

DOI : $10.4000 /$ clio. 9403

ISSN : 1777-5299

Éditeur

Belin

Édition imprimée

Date de publication : 15 décembre 2009

Pagination : 99-117

ISSN : $1252-7017$

Référence électronique

Mercedes Yusta, «Vierges guerrières et mères courage : le panthéon des communistes espagnoles en exil », Clio. Histoire, femmes et sociétés [En ligne], 30 | 2009, mis en ligne le 15 décembre 2012, consulté le 01 mai 2019. URL : http://journals.openedition.org/clio/9403; DOI : 10.4000/clio.9403

Tous droits réservés 


\section{Vierges guerrières et mères courage : le panthéon des communistes espagnoles en exil}

Mercedes YUSTA

Rien ne fait fléchir ces héroïnes qui placent par-dessus tout le nom de l'Espagne et de ses femmes. Les tortures qu'on leur fait subir sont telles que l'on frémit à leurs récits. Mais il faut les lire pour que nous n'oubliions jamais (Mujeres Antifascistas Españolas, 1, novembre 1946).

La fin de la guerre civile espagnole (1936-1939) provoqua un exil sans précédent dans l'histoire de l'Espagne. Des centaines de milliers de personnes traversèrent la frontière franco-espagnole, notamment en février 1939, après la conquête de la Catalogne par les troupes de Franco. Parqués par les autorités de la III République dans des camps de réfugiés, hommes et femmes, militants des partis et des organisations appartenant au Front populaire, allaient affronter la politique de Vichy contre les «étrangers indésirables » et l'occupation allemande. En dépit de ces circonstances, les républicain-e-s espagnol-e-s réussirent à créer des organisations, comme l'Union Nationale Espagnole où s'organisa leur participation à la Résistance ${ }^{1}$. Or, en dépit du nombre important d'associations féminines républicaines, seules les femmes liées au Parti communiste espagnol (PCE) réussirent à reconstruire l'association des Mujeres Antifascistas, la plus importante des organisations féminines espagnoles des années 1930². L'Union des Femmes Françaises (UFF), liée au PCF, les aida à remettre sur pied leur organisation en France.

Quelques mois après la Libération, en mars 1945, un groupe de militantes, dont la responsable du PCE en France, Carmen de Pedro,

1 Dreyfus-Armand 1999.

2 Nash 1981, 1999. Sur l'organisation des femmes antifascistes espagnoles en exil voir Nadal 1989, Dominguez 1994, Yusta 2009. 
se réunit à Toulouse pour réorganiser «légalement» leur «ancienne organisation de Femmes Antifascistes en lui donnant le nom d'Union des Femmes Espagnoles $»^{3}$. Le retour de la dirigeante communiste Dolores Ibárruri, La Pasionaria, de son exil soviétique, où elle avait passé la guerre, marque un tournant avec la reprise en main par la direction communiste des structures du Parti et de l'organisation féminine. Ce fut la femme de confiance de Dolores Ibárruri, sa secrétaire Irene Falcón, qui prit la tête de l'organisation lors de son premier Congrès réuni en août 1946, alors que la Pasionaria y était désignée présidente honoraire. Dès lors, l'organisation féminine allait devenir un puissant instrument d'encadrement des femmes républicaines en exil jusqu'à sa dissolution en septembre 1950, à la suite de l'opération menée par la police française visant à mettre hors la loi toute organisation communiste étrangère sur le sol français.

\section{Poursuivre le combat contre le régime franquiste}

Parmi les objectifs de l'Union des femmes espagnoles, le plus essentiel était de continuer la lutte contre la dictature de Franco par tous les moyens possibles, principalement en soutenant la résistance intérieure. Cette mission s'appuyait sur une stratégie, des tâches et des moyens spécifiques aux femmes. Par ailleurs, une grande énergie fut mobilisée pour produire un discours justifiant l'action politique des femmes dans l'espace public et l'importance de leur rôle au sein de l'antifranquisme, en particulier à travers une publication mensuelle, Mujeres Antifascistas Españolas, parue de novembre 1946 à septembre 1950, date de l'interdiction du PCE et de ses organisations satellites. Dans ses pages, l'organisation des femmes proposait aux exilées un cadre, à la fois culturel et politique, à l'intérieur duquel celles-ci pouvaient insérer leur expérience et leur pratique militante.

Même si elle naît en exil, l'Union des Femmes Espagnoles ne se définit pas comme une organisation de femmes exilées mais «se considère partie intégrante de cette hérö̈que organisation des Femmes Antifascistes d'Espagne $»^{4}$. Elle revendique alors un double

3 Marina García, «Informe general », Unión de Mujeres, boletín interior, 9, Toulouse, août 1946.

4 Mundo Obrero, 1 de agosto de 1946. 
enracinement : chronologique, puisqu'elle se situe dans la continuité de l'organisation féminine des années 1930; et géographique, puisqu'elle se présente comme une grande organisation de femmes antifascistes qui s'étendrait des deux côtés de la frontière. Il faut dire que l'organisation de l'intérieur, qualifiée d'«héroïque», avait une existence plutôt précaire, voire fantasmatique: la répression en Espagne ne permettait pas vraiment la reconstitution de réseaux, mais plutôt de petits groupes de femmes, plus ou moins isolés, qui essayaient d'accomplir certaines tâches indispensables, comme le secours aux prisonniers politiques ou la distribution de tracts. Mais les femmes de l'exil leur accordent une place très importante dans leur presse, puisque l'activité de la résistance intérieure, aussi minimale fût-elle dans un premier temps, était le gage de la continuité de la lutte entamée en 1936, et donc de la résistance du peuple espagnol contre le fascisme ${ }^{5}$.

Autour de cette lutte se construit alors, dans la revue des femmes communistes, un récit qui retrace les contours du combat antifasciste né pendant la Seconde République et dont les protagonistes sont principalement des femmes. Il y a des « héroïnes » clairement identifiées et régulièrement évoquées; la liste de leurs noms constitue une sorte de litanie qui construit un véritable "panthéon» féminin ${ }^{6}$. Mais on trouve aussi des héroïnes anonymes, en général des femmes de l'intérieur, mortes des suites de la répression, dont l'histoire est racontée dans des récits qui, du fait des mécanismes narratifs employés, relèvent davantage de la fiction littéraire que d'un récit objectif. Ces histoires, dont la vraisemblance ne fait pas de doute, ne peuvent cependant être qualifiées de «vraies»: il s'agit plutôt de construire, à partir de données empruntées à la réalité et en utilisant des mécanismes de fictionnalisation ${ }^{7}$, des récits exemplaires qui ont pour sujet la résistance des femmes et la répression terrible à laquelle elles sont soumises. Plutôt qu'à des femmes identifiables, ces récits renvoient à des archétypes féminins, comme la vierge guerrière ou la mère héroïque. C'est-à-dire, à des « héroïnes » archétypiques à travers

5 Yusta 2004

6 J'emprunte cette expression à Dermenjian 2004.

7 Iser 1997. 
lesquelles se transmettent une histoire, une mémoire, une morale et une éthique collectives qui soudent ainsi les exilées autour d'une culture politique partagée.

\section{Le culte des héroïnes : vierges guerrières et mères courage}

Ce culte des héroïnes est un élément constitutif de la culture politique de l'organisation des Espagnoles en exil, comme ce fut d'ailleurs le cas pour d'autres organisations communistes. Ce «culte » concerne de manière presque exclusive des personnages féminins et d'ailleurs il a été construit, promu et appliqué de manière tout aussi exclusive par l'organisation des femmes, émanation féminine du Parti. Il est intéressant de noter que tel est aussi le cas des militantes de l'Union des Femmes Françaises (UFF), organisation sœur des Espagnoles, dont celles-ci se sont largement inspirées pour élaborer aussi bien leur discours que leurs pratiques. L'exception à cette règle serait la figure de Dolores Ibárruri, La Pasionaria. Son "culte», répandu aussi bien parmi les femmes que parmi les hommes communistes, relève à la fois d'un culte de la personnalité typique de certaines organisations communistes et d'un processus d'héroïsation ${ }^{8}$. Curieusement, ces processus d'héroïsation sont très rares en ce qui concerne les hommes, hormis certains guérilleros victimes de la répression franquiste, comme Cristino García ou Agustín Zoroa. Et surtout, ces héros ne semblent pas avoir été l'objet d'un "culte» comparable à celui qui entoure les femmes, ce qui est probablement à mettre en relation avec l'histoire interne du PCE. En effet, pendant cette période, les règlements de comptes et les épurations furent courants parmi les cadres masculins du Parti. Les femmes, éloignées des postes à responsabilité, à l'exception notable de la Pasionaria, étaient nettement moins controversées et il était plus aisé d'en faire des héroïnes.

Sandra Fayolle a étudié les implications de ces processus d'hérö̈sation pour l'UFF. Elle note à ce propos que s'y «sont progressivement construites une mémoire des lieux et des formes de commémoration particuliers, mais aussi tout un ensemble de

8 Cruz 1999, Ripa 1997, Avilés 2005. Sur la sociologie de la mémoire communiste voir Lavabre 1994. 
référents culturels communs $»^{9}$. Les mêmes observations sont applicables aux femmes de l'Union des femmes espagnoles. Mais, alors que dans l'UFF les manifestations du « culte des héroïnes » sont concentrées sur la figure de Danielle Casanova, résistante morte en déportation, les Espagnoles ont construit un véritable «panthéon» mémoriel collectif, où plusieurs figures féminines sont commémorées et honorées. Il s'agit, en général, de jeunes militantes mortes, soit au cours des combats, soit des suites de la répression. Leurs noms sont égrenés dans les pages de la revue et accompagnés d'injonctions au souvenir et à la reconnaissance. La revue accomplit également un travail didactique, notamment à travers la chronique intitulée «Nos héroïnes » qui les érige en exemple et en modèle.

La liste des héroïnes espagnoles s'ouvre habituellement, selon une logique chronologique, par les noms de deux jeunes filles, Aïda Lafuente et Lina Odena, que la revue qualifie de premières «martyres» de l'organisation des femmes. La première militante tomba en 1934, lors de la «Révolution d'Octobre » (sic) qui opposa, aux Asturies, des mineurs socialistes et communistes au gouvernement de droite d'Alejandro Lerroux. Aïda Lafuente mourut, au pied de sa mitrailleuse, lors de la prise d'Oviedo et devint une figure paradigmatique de la «vierge guerrière ». Les données historiques sur cette femme sont rares et confuses ; pour certains, elle aurait été fusillée après avoir été capturée, pour d'autres, elle aurait été assassinée sur place à côté de sa mitrailleuse. Pour d'autres encore, elle serait morte lors de l'assaut des troupes de légionnaires envoyées par le gouvernement pour écraser la révolte des Asturies. Il est presque certain, par ailleurs, qu'elle était une militante libertaire et qu'elle avait 19 ans, alors que les différents récits ont tendance à la présenter comme une très jeune fille de 15 ou 16 ans. En tout cas, sa mythification intervient presque immédiatement après les événements et, déjà pendant la guerre civile, son nom est évoqué par les dirigeants du Front populaire comme un modèle d'héroïsme au féminin ${ }^{10}$.

Lina Odena, jeune dirigeante communiste qui devint secrétaire générale de l'organisation des femmes après la victoire du Front

\footnotetext{
$9 \quad$ Fayolle $2003: 368$.

10 Bunk 2003, 2007.
} 
populaire, appartient au même type d'hérö̈ne virginale. Au cours d'un contrôle franquiste, au tout début de la guerre civile, elle préféra se donner la mort avec son pistolet plutôt que de tomber entre les mains des phalangistes : le récit de sa mort l'apparente aux femmes martyres de la tradition chrétienne qui préférèrent la mort au déshonneur, ici représenté par les tortures et sévices, notamment à caractère sexuel, auxquelles étaient souvent soumises les femmes "rouges» par les franquistes ${ }^{11}$. Ce n'est d'ailleurs pas le seul cas de suicide parmi les femmes de ce panthéon. L'avocate communiste Matilde Landa, prisonnière dans la prison de Ventas, se suicida en 1942, dans des circonstances qui ne sont pas totalement éclaircies mais qui auraient à voir avec les pressions auxquelles elle fut soumise pour la forcer à se convertir au catholicisme : son nom apparaît aussi régulièrement dans les listes des héroïnes « dont le peuple doit se souvenir $»^{12}$.

Dans le registre des "héroïnes virginales", une place toute particulière est accordée à un groupe de treize jeunes femmes, dont certaines mineures, exécutées à Madrid juste après la fin de la guerre. L'histoire de ces jeunes femmes, évoquées sous le nom collectif des "treize roses », a connu une longue postérité jusqu'à nos jours avec la récente parution d'un livre et d'un film qui leur sont consacrés ${ }^{13}$. Or, à ma connaissance, la revue Mujeres Antifascistas Españolas est la première publication à évoquer le cas de ces militantes des Jeunesses socialistes unifiées incarcérées juste après la guerre, et à donner les principaux éléments qui ont servi à construire ensuite le récit mythifié de leur mort. D'ailleurs la revue en parle déjà dans son premier numéro de 1946 : seuls deux noms, celui de Concha Conesa (qui s'appelait en réalité Julia Conesa) et «Blanquita » (Blanca Brisac) sont alors connus des femmes qui rédigent les articles de Mujeres Antifascistas Españolas. Ce sont ces deux noms qui intègreront le «panthéon» collectif des héroïnes antifascistes, alors qu'il faut attendre le dernier numéro de la revue, celui de septembre 1950, pour trouver un récit complet du sort réservé à ces jeunes femmes.

\footnotetext{
11 Joly 2008.

12 Ginard 2005.

13 Il s'agit du film d'Emilio Martínez Lázaro, Las trece rosas (Espagne, 2007) et du livre de Carlos Fonseca 2004. Voir à ce propos Céspedes 2007.
} 
D'autres femmes, vivantes cette fois-ci, furent qualifiées d'« héroïnes » et devinrent de véritables saintes laïques. Il s'agit de Mercedes Gómez, Isabel Sanz Toledano et Teresa Toral, militantes communistes incarcérées dans les prisons franquistes après la fin de la guerre, qui y risquaient la peine de mort. Leur évocation dans la revue et leur qualification d'héroïnes de l'antifranquisme avait un sens pragmatique immédiat: il s'agissait de sensibiliser l'opinion publique internationale sur le sort de ces femmes et, par une large campagne de protestation, d'éviter qu'elles ne fussent exécutées par la dictature. Finalement, cette campagne fut efficace et elles échappèrent au sort funeste des autres «héroïnes». Ainsi, contrairement aux autres femmes citées ici, elles n'entrèrent pas durablement au panthéon des héroïnes antifascistes et la mémoire historique des femmes communistes n'a pas retenu leurs noms.

\section{Mémoire nationale et réécriture de l'histoire}

Aïda Lafuente et Lina Odena sont deux cas particuliers dans la formation $\mathrm{du}$ panthéon féminin antifranquiste, puisque leur héroïsation et la célébration de leur mémoire commencent déjà pendant la guerre civile. Par ailleurs, elles sont célébrées par tout le spectre de la gauche républicaine et pas seulement par les communistes. Leur hérö̈sation est caractéristique d'un processus commun aux deux camps qui s'affrontent durant la guerre civile, celui de la réappropriation et de la réécriture de l'histoire pour exclure l'ennemi de l'histoire nationale. Ce processus est particulièrement marqué dans le camp franquiste, qui s'auto-qualifie de camp «national», mais il est visible aussi dans le camp républicain. Les deux camps se disputent ainsi les symboles de l'identité nationale et en inventent de nouveaux. L'« espagnolité », revendiquée par les franquistes et les républicains, se revêt d'un caractère idéologique conforme au projet politique de chacun des protagonistes du conflit ${ }^{14}$.

Dans le combat idéologique pour la redéfinition de l'identité nationale qui se prolongera bien après la fin de la guerre, les héroïnes

14 Nuñez Seixas 2006. Pour les processus de réécriture de l'histoire dans l'Espagne contemporaine, voir aussi Boyd 2000. 
jouent un rôle particulier dans le camp républicain. Des femmes comme Aïda Lafuente et Lina Odena appartiennent au patrimoine historique des Espagnol-e-s et s'inscrivent ainsi dans un continum historique qui permet de retracer les luttes pour la liberté, dont la guerre civile, puis la résistance antifranquiste, seraient les derniers avatars. Ainsi se construit, pendant la guerre, une séquence historique bien définie reprise par la suite en exil. La séquence démarre avec la lutte contre les troupes napoléoniennes au début du XIX ${ }^{\mathrm{e}}$ siècle, lors de la guerre «d'Indépendance» (1808-1814). Elle se poursuit avec la lutte des libéraux contre le dernier représentant de l'absolutisme, Ferdinand VII (1814-1833); ensuite un grand saut chronologique mène à la Révolution des Asturies contre le gouvernement de droite d'Alexandre Lerroux (1934), à la guerre civile (1936-1939) et à la résistance antifranquiste.

La généalogie des héroïnes (républicaines et antifascistes d'abord, antifranquistes ensuite) suit cette séquence historique. Pendant la guerre, dans une affiche du Secours Rouge de Valencia ${ }^{15}$, on peut lire les noms d'Agustina de Aragón, Mariana Pineda, Aïda Lafuente et Lina Odena, avec la légende: "Héroïnes de la liberté et de l'indépendance de l'Espagne ». La première a participé à la Guerre de l'Indépendance, la deuxième à la lutte contre l'absolutisme de Ferdinand VII. Déjà incluses, dès le XIX ${ }^{\mathrm{e}}$ siècle, dans un processus d'héroïsation, elles préfigurent les femmes combattantes et résistantes du XXe siècle. Agustina de Aragón s'empara d'un canon qu'elle dirigea contre les troupes napoléoniennes lors du siège de Saragosse en 1808 ; Mariana Pineda appartenait à un réseau libéral clandestin et fut exécutée publiquement en 1831 pour avoir brodé un drapeau libéral. Agustina de Aragón est la plus ambigüe des deux, du point de vue politique, puisqu'elle s'est battue contre les Français à la fois occupants étrangers, mais aussi représentants du progrès et les Lumières. Sa figure est reprise et revendiquée par les deux camps. Pour les Franquistes, elle représente la défense de la Patrie menacée par les forces étrangères ; les Républicains en feront une icône de la résistance féminine face à l'oppression, une ancêtre, en quelque sorte, des miliciennes antifascistes dont le rôle était cependant controversé :

15 Voir le Cabier d'images, figure 2. 
rappelons qu'elles avaient été rapidement expulsées du front, notamment par les communistes qui défendaient la formation d'une armée régulière et se méfiaient de l'indépendance des milices.

Pour les femmes en exil, Agustina de Aragón devient une icône qui assure la jonction entre les héroïnes du passé et celles du présent. De façon plus générale, la Guerre de l'Indépendance devient, pendant la guerre civile et l'après-guerre, le symbole de la lutte du peuple espagnol contre l'oppression et offre des exemples de femmes qui ont lutté pour la défense de la Patrie. Ainsi, en mai 1947, la commémoration des journées de mai 1808 où le peuple madrilène se révolta contre les occupants français, donne lieu à la réaffirmation de ce continum historique : «1808-1936-1947 : aujourd'hui comme hier les femmes espagnoles luttent pour l'indépendance de la Patrie $»^{16}$. En 1948, la même commémoration est l'occasion de proclamer que les femmes de Madrid qui résistent contre la dictature sont les «dignes héritières de l'amour patriotique des Madrilènes $»^{17}$. On célèbre de nouvelles «Agustina de Aragón», des femmes du peuple qui réincarnent cet héroïsme populaire, comme Manuela Sánchez, paysanne galicienne et collaboratrice de la guérilla antifranquiste, assassinée par la Garde Civile franquiste :

MANUELA SÁNCHEZ, fille et Galice et de l'Espagne immortelle, dans tes veines courait le sang de Maria Pita, d'Agustina de Aragón, de Vilaboy, de Cristino, de Manuel Ponte, des héros d'hier, d'aujourd'hui et de toujours; des héros du peuple. Les chiens fascistes ont brûlé ton corps, ont détruit les tiens, mais ta geste d'épopée (sic) vivra éternellement dans le cœur de la Patrie ${ }^{18 .}$

Ces héroïnes ont une double fonction, à la fois mémorielle et identitaire. Proposées comme exemples et comme modèles, elles représentent les valeurs politiques, éthiques et morales que véhicule l'organisation. Elles sont aussi les derniers maillons d'une généalogie féminine qui unit les femmes du présent aux héroïnes du passé. Ces invocations rappellent très fortement les cultes à caractère religieux,

\footnotetext{
16 Mujeres Antifascistas Españolas, 8, mai 1947, p. 6.

17 Mujeres Antifascistas Españolas, 20, mai 1948, p. 6.

18 «Manuela Sánchez, heroina de España », Mujeres Antifascistas Españolas, 22, juillet 1948, p. 15-16.
} 
en particulier catholiques, auxquels elles se substituent en quelque sorte. Les invocations aux «martyres » républicaines font ainsi écho à celles des «martyrs tombés pour Dieu et pour l'Espagne» de l'Espagne franquiste, dont le culte renvoie sans ambiguïté à une transcendance religieuse. Pour les hérö̈nes antifascistes, un autre type de transcendance renvoie à l'histoire et à la mémoire des combats collectifs. Ces femmes martyrisées deviennent ainsi les symboles d'un peuple qui ne meurt jamais.

\section{Entre mythe et réalité}

Au fil des pages de la revue Mujeres Antifascistas Españolas, un culte se met en place à travers la remémoration et la répétition des noms des héroïnes, mais aussi à travers la transmission de récits qui reviennent sur les plus connues ou donnent des informations sur celles qui résistent en Espagne. La revue consacre un espace très important à des témoignages, des lettres et des documents qui font état de luttes menées par les Espagnoles antifascistes contre la dictature, ainsi que de la terrible répression dont elles sont victimes.

La forme que prennent ces témoignages est intéressante dans la mesure où ils sont souvent réécrits par les rédactrices. Ainsi, tout en étant inspirés de faits réels, les récits prennent la forme de véritables nouvelles, avec des personnages - presque toujours des femmes, le plus souvent anonymes - dont le statut est ambigu, entre personnage historique et archétype littéraire, entre mythe et réalité. Ces histoires deviennent ainsi des récits d'édification, de véritables paraboles ou contes moraux qui présentent d'étroits liens de parenté avec la littérature espagnole ouvrière de fiction du premier tiers du $\mathrm{XX}^{\mathrm{e}}$ siècle ${ }^{19}$.

Le mythe des «treize roses » permet de montrer les mécanismes à l'œuvre dans ce système d'héroïsation/fictionnalisation. L'histoire de ces jeunes filles est évoquée à deux reprises, de façon sommaire, dans le premier numéro de la revue et de façon beaucoup plus détaillée dans le dernier, le numéro 39. Quatre ans séparent donc ces deux versions de l'histoire. En 1946, le qualificatif de «treize roses»

19 Magnien 1995. 
n’apparaît pas encore. La revue se limite, dans ce premier numéro, à reproduire le témoignage d'une jeune femme, rescapée des prisons franquistes, qui aurait connu les jeunes filles:

J'ai vu sortir un jour treize filles pour être jugées parce qu'elles avaient récolté de l'argent pour les prisonniers..

On les condamna à mort...

On leur nia la possibilité de faire appel et elles furent fusillées à côté de 50 garçons, aussi jeunes qu'elles, devant les murs du Cimetière de l'Est.

Ils demandèrent à être fusillés tous ensemble, mais on ne leur accorda pas ce vœu. La Phalange ne pouvait supporter que les jeunes hommes et les jeunes filles, dans un dernier cri, lançassent au monde leur confiance des lendemains de lumière et de liberté pour leur Patrie ${ }^{20}$.

Certains éléments de cette narration sommaire seront repris par la suite dans différentes versions, en particulier dans le récit d'une mort héroïque publié en 1950. Le sobre récit à la première personne de 1946 laisse la place à une reconstruction littéraire fournissant de nombreux détails impossibles à connaître de première main. Les principaux éléments narratifs qui construisent le mythe des «treize roses » apparaissent pleinement développés dans cette version de 1950 : l'activité clandestine des filles dans la JSU, leur arrestation suite à la "délation d'une moucharde ", la prison, la condamnation à mort. La sérénité avec laquelle les «treize roses» affrontent la mort, les préparatifs qu'elles font avant de mourir, font penser à une version laïque des histoires des premiers martyrs chrétiens si abondantes dans les livres pieux de l'époque :

Elles sont allées se doucher, se sont coiffées, ont mis leurs meilleurs vêtements. Conchita Conesa sortit une bague, souvenir de famille, et la donna à sa meilleure amie. Blanquita a insisté pour qu'on lui coupe sa longue tresse noire pour l'envoyer à sa famille [...] Elles ont donné d'ultimes conseils :

- Ne faiblissez pas dans la lutte. Dites à ceux qui sont dehors de s'organiser, de continuer. La seule chose que nous regrettons c'est de n'avoir pu continuer avec notre travail...

20 «...Y llegaron a nosotras... », Mujeres Antifascistas Españolas, 1, 1 ${ }^{\mathrm{er}}$ novembre 1946, p. 3. 
[...] Droites, souriantes, elles marchèrent vers la mort avec un «Vive la République! » aux lèvres.

[...] Le 5 août 1939 les treize roses, symbole de la jeunesse espagnole qui préfère mourir plutôt que de plier devant l'ennemi, ont écrit avec leur sang un des premiers chapitres de la lutte héroïque et silencieuse du peuple espagnol sous l'oppression franquiste ${ }^{21}$.

Les fonctions de ce type de récits sont multiples. Il s'agit de dénoncer, de provoquer le refus et l'indignation, mais surtout de fonder, par l'émotion et l'identification à ces images fortes, une mémoire collective faite d'épisodes fondateurs. Il s'agit de ce que Marie-Claire Lavabre appelle une "mémoire historique ", c'est-à-dire un récit instrumentalisé du passé qui sert à conforter la culture politique et l'identité du groupe ${ }^{22}$. Les treize roses deviennent ainsi des paradigmes du combattant, ou plutôt de la combattante antifranquiste: courageuse, solidaire, sans peur de la mort, se projetant toujours sur la lutte collective du peuple, dans une sorte de transcendance qui permet de dépasser la mort individuelle pour s'incarner dans une instance collective, le peuple antifranquiste. L'écrivain communiste Jesús Yzcaray, qui excella dans l'écriture de ce genre de récits oscillant entre réalité et fiction, l'exprima encore plus clairement en plaçant dans la bouche d'un guérillero antifranquiste la formule suivante: "Je suis peuple, et pour le peuple il n'y a pas de mort $»^{23}$.

L'histoire des treize roses renvoie à un événement et à des femmes qui ont réellement existé. Or, dans les pages de la revue Mujeres Antifascistas Españolas apparaissent d'autres récits dont le statut est bien plus problématique, puisque leur nature, réelle ou fictive, n'est jamais clairement établie. C'est le cas, entre autres, d'un récit écrit par la journaliste Rosa Vilas (auteure, par ailleurs, de la plupart des récits publiés dans la revue), intitulé «Je veux être guérillera $»^{24}$.

21 «Las trece rosas», Mujeres Antifascistas Españolas, 39, septembre-octobre 1950, p. 11

22 Lavabre 1994 : 15-19.

23 Jesús Yzcaray, «30 días con los guerrilleros de Levante. VI. ...Y en su bandera está escrito », Mundo Obrero, 10 juin 1948, p. 3.

24 Rosa Vilas, "Yo quiero ser guerrillera », Mujeres Antifascistas Españolas, 19, mars 1948 , p. 8. 
Le récit raconte l'histoire de Carmeta, jeune fille incarnant à la perfection le modèle de l'héroïne virginale. Détenue à cause de sa parenté avec un guérillero, Carmeta risque d'être « interrogée » par un phalangiste à la «bave visqueuse» : devant la menace à peine voilée de viol, Carmeta réussit à tuer son agresseur et à rejoindre la guérilla. Face aux héroïnes sacrificielles, face aux suicides des héroïnes réelles (Matilde Landa, Lina Odena), la revue propose ainsi aussi à ses lectrices un modèle d'héroïne de fiction qui prend son destin en main et échappe ainsi à un sort - le viol, le déshonneur - qui, dans la culture traditionnelle espagnole, a toujours été considéré comme pire que la mort.

\section{La maternité héroïque}

Les considérations sur l'« honneur» féminin et la virginité et la place qu'ils semblent encore occuper dans l'imaginaire collectif des femmes antifascistes suggère que ces femmes (et la culture politique communiste de manière générale) ne mettent pas en question le système de genre traditionnel25. En même temps, il est évident qu'elles revendiquent la citoyenneté dont les femmes jouissaient lors de la Seconde République et qu'elles légitiment la présence des femmes dans l'espace public par leur rôle de première importance dans la lutte antifranquiste. Mais la jonction entre ces deux aspects reste problématique : la logique de l'émancipation féminine ne devaitelle pas conduire à remettre en cause une répartition des rôles où les femmes étaient souvent cantonnées à la gestion de l'espace privé ?

Les femmes de l'organisation antifasciste surmontent cette contradiction à travers une exaltation de la maternité, source d'autorité et de légitimité pour intervenir aussi dans l'espace public. Il s'agit là d'un trait commun à toutes les organisations féminines communistes, comme l'UFF ou la Fédération démocratique internationale des femmes qui renvoie à l'idéologie familialiste développée par les partis communistes dès les années 1930. Mais ce maternalisme s'accentue pendant les années de la guerre froide, avec la concurrence, notamment en France, entre organisations féminines

25 Nash 1999 ; Yusta 2009. 
catholiques et communistes pour le contrôle des «masses féminines $»^{26}$. Mais dans le cas des Espagnoles, cela renvoie également à une stratégie déjà mise en place pendant la guerre civile qui consiste à utiliser les références à la maternité comme un discours mobilisateur adressé aux femmes. L'autorité des mères est invoquée dans l'espace public comme un argument pour justifier la participation féminine aux débats et combats politiques ${ }^{27}$.

Pendant la guerre civile, l'organisation des femmes en appelle constamment aux sentiments maternels pour convaincre les femmes de la nécessité de s'engager dans le combat antifasciste. Apparaît ainsi la figure de la «mère combative » dont parle Mary Nash; une figure qui a l'avantage de remplacer la sulfureuse miliciana comme modèle d'identification. En effet, la mère combative participe à l'effort de guerre en projetant ses vertus maternelles dans l'espace public: le soin, l'attention aux plus faibles, la recherche du bien-être collectif. En ce sens, elle incarne parfaitement la répartition des rôles préconisée par les communistes pendant la guerre et popularisée par la Pasionaria elle-même avec le mot d'ordre «Les hommes au front, les femmes à l'arrière-garde », totalement compatible avec un système de genre traditionnel ${ }^{28}$. Dans le contexte de l'exil, la mère combative de la guerre civile deviendra la mère résistante antifranquiste.

Une fois de plus, les récits qui mettent en scène ces mères antifranquistes, héroïques par définition, laissent souvent planer l'ambiguïté par rapport à leur existence réelle. Ce qui importe, en fait, c'est l'enseignement moral transmis à travers ces histoires. Ainsi, dans le récit "Mère de guérillero », Rosa Vilas met en scène une femme du peuple âgée, dont l'histoire est qualifiée de « véridique » mais dont le nom ou toute autre donnée permettant de l'identifier sont absents du texte. L'histoire met en scène le sacrifice suprême de cette mère, qui se laisse torturer à mort avant d'avouer où se cache son fils guérillero. Le caractère véridique du récit est finalement secondaire : il s'agit de représenter, par le biais d'une histoire individuelle, l'hérö̈sme suprême que l'on attribue aux résistantes. Il importe aussi d'incarner,

\footnotetext{
26 Chaperon 2000 ; Trat 2002 ; Yusta 2009.

27 Nash 1999.

28 Nash 1999 ; Ripa 1997.
} 
à travers une histoire exemplaire, les innombrables victimes de la répression énumérées dans les revues de l'exil. Ainsi, la femme du récit représente en réalité une foule d'héroïnes sans nom, et le récit se ferme sur une injonction au souvenir de ces victimes anonymes: "Quand, dans les listes des martyrs que les sbires fascistes ont froidement assassinés, vous lirez : 'à Galice, une paysanne', pensez à cette femme courageuse $»^{29}$.

Or, la femme qui incarna à la perfection ce modèle de mère antifranquiste fut Dolores Ibárruri elle-même. Comme Jeannette Vermeersch dans le cas du PCF et de l'UFF, Dolores Ibárruri fut l'objet d'un véritable culte de la personnalité qui, étant donné l'autorité de cette femme dans le PCE, sa renommée internationale et le mythe qui s'était déjà forgé sur elle pendant la guerre, alla beaucoup plus loin que celui de son homologue française ${ }^{30}$. La revue Mujeres Antifascistas Españolas construit, avec Dolores Ibárruri, un personnage hérö̈que, à la fois mythique et réel, qui devint la parfaite incarnation des idéaux de l'organisation. Dolores est la dirigeante, la militante, mais par-dessus tout, elle est la mère. La mère qui a accompli le sacrifice suprême, celui de la mort du fils bien-aimé, puisque Rubén Ruiz, le fils de Pasionaria, est tombé pendant le siège de Stalingrad. Ainsi, Dolores Ibárruri devint elle-même l'archétype de la mère "généreuse et héroïque » (comme l'a dit Irene Falcón), tout en étant aussi une femme politique de premier plan ${ }^{31}$. Les femmes de l'Union des Femmes Espagnoles s'appuieront sur leur condition de mères, réelles ou potentielles, pour faire entendre leurs voix et pour porter dans l'espace public la dénonciation de la dictature. La situation de pénurie vécue dans l'Espagne franquiste, avec son corollaire de maladies et de mortalité infantile, devait ainsi donner des raisons suffisantes aux femmes pour s'engager dans la lutte antifranquiste :

La douleur des mères qui voient mourir leurs enfants parce que manquent le lait, le pain et les remèdes que réclament leurs pauvres petits

29 Rosa Vilas, « Madre de guerrillero », Mujeres Antifascistas Españolas, 15, novembre 1947 , p. 8.

$30 \quad$ Rousseau 1983 ; Ripa 1997 ; Cruz 1999 ; Avilés 2005.

31 Irene Falcón, « Pasionaria en Checoeslovaquia », Mujeres Antifascistas Españolas, 4, 15 janvier 1947, p. 7. 
corps, se transforme en haine redoutable contre les responsables de cette situation tragique: Franco et son régime criminel. Et cette haine est à son tour un puissant élan pour l'action antifranquiste (...) qui touche déjà des milliers de femmes ouvrières et paysannes, des employées et des intellectuelles, des femmes au foyer, des mères de famille, qu'elles soient jeunes ou âgées ${ }^{32}$.

Cette rhétorique est commune à celle des autres mouvements féminins européens de gauche, notamment la Fédération démocratique internationale des femmes et l'UFF. Dans le contexte de la Guerre froide, ces organisations sont engagées dans un combat pacifiste qui fait de l'Union soviétique le pays défenseur de la paix face aux «puissances impérialistes », les États-Unis et leurs alliés. Les Espagnoles doivent dès lors combiner la lutte antifranquiste et les stratégies de ces autres organisations féminines, dont l'appui leur est indispensable pour rendre visible la lutte contre la dictature de Franco dans le contexte de l'exil ${ }^{33}$.

Les adhérentes de l'Union des Femmes Espagnoles sont restées longtemps attachées à une rhétorique de l'héroïsme et du combat éloignée du discours pacifiste des organisations féminines européennes. Une formule leur permet de faire la jonction entre cette lutte pour la paix où elles prennent place en tant que mères et la lutte que leurs héros et héroïnes de l'intérieur mènent contre la dictature : «Franco, c'est la guerre ${ }^{34}$. Lutter contre la dictature, même les armes à la main, revient donc à lutter pour la paix : vierges guerrières et mères courage peuvent continuer à être célébrées comme des combattantes engagées dans une double lutte, contre la dictature franquiste et pour la paix mondiale. Même si, finalement, ces figures héroïques renvoient toujours les femmes de l'Union des femmes espagnoles à un autre et à un ailleurs : hormis Dolores Ibárruri, modèle par définition inaccessible, il n'y a pas d'héroïne de l'exil.

\footnotetext{
32 «Jornada internacional de la mujer : ¡España no será carne de cañón de los imperialistas angloamericanos! », Mujeres Antifascistas Españolas, 35, mars 1950.

33 Yusta 2009.

34 «El franquismo es una amenaza para la paz », Mujeres Antifascistas Españolas, 28, juin 1949, p. 2.
} 
Ainsi, le culte développé autour des héroïnes contribue à maintenir les femmes de l'exil dans un imaginaire qui les rattache à l'Espagne républicaine, à un temps et à un pays à jamais disparus et devenus, à leur tour, mythiques.

\section{Bibliographie}

AlCón Eva, 2006, "Resistencia y compromiso de las mujeres antifascistas », Asparkía. Investigació feminista, 17, p. 141-164.

Alted Alicia, 1997, «El exilio republicano español de 1939 desde la perspectiva de las mujeres ", Arenal. Revista de Historia de Mujeres, 4/2, p. 223-238.

Andreu Xavier, Alcolea Àngels \& Pilar Molina, 2001, «El doble exili de les dones del 39 ", in Fernanda Mancebo, Marc BALdò \& Cecilio Alonso (ed.) L'exili cultural de 1939. Seixanta anys després. Actas del I Congreso Internacional (Valencia, 2001). T. 2, Universitat de València/Biblioteca Valenciana, p. 81-91.

Avilés Juan, 2005, Pasionaria, la mujer y el mito, Barcelona, Plaza \& Janés.

Berkovitch Nitza, 1999, From Motherhood to Citizenship. Women's Rights and International Organizations, Baltimore \& London, The John Hopkins University Press.

BoyD Carolyn, 2000, Historia Patria. Politica, historia e identidad nacional en españa: 1875 1975. Pomares-Corredor, Barcelona.

Bunk Brian D., 2003, « Revolutionary warrior and gendered icon: Aida Lafuente and the Spanish Revolution of 1934 », Journal of Women's History, 15/2, p. 99-122.

—, 2007, Ghosts of Passion: Martyrdom, Gender, and the Origins of the Spanish Civil War, Durham, Duke University Press.

Bussy Genevois Danièle, 1990, «Les Espagnoles ou le pacifisme de l'entre-troisguerres », in Rita THALMANN (dir.), La tentation nationaliste, 1814-1945, Paris, Tierce-CNRS, p. 115-135.

CÉSPEDES Jaime, 2007, « Las trece rosas de la guerra civil vistas por el novelista Jesús Ferrero y el periodista Carlos Fonseca », Tonos. Revista electrónica de estudios filológicos, 14. http://www.um.es/tonosdigital/znum14/secciones/estudios-413rosas.htm\#_ftnref18.

Chaperon Sylvie, 2000, Les années Beawvoir (1945-1970), Paris, Fayard.

Cruz Rafael, 1999, Pasionaria. Dolores Ibárruri, Historia y Simbolo, Madrid, Biblioteca Nueva.

Dermenjian Geneviève, Lapied Martine \& Jacques Guilhaumou (dir.), 2004, Le Panthéon des femmes: Pratiques et représentations des héroïnes, Paris, Publisud. 
Domínguez Pilar, 1994, Voces del exilio. Mujeres españolas en México 1939-1950. Comunidad de Madrid-Dirección General de la Mujer, Madrid.

Dreyfus-Armand Geneviève, 1999, L'exil des républicains espagnols en France. De la guerre civile à la mort de Franco, Albin Michel, Paris.

DuCHEN Claire \& Irène BANDHAuER SCHOFFMAN (eds), 2000, When the War was over: Women, War and Peace in Europe, 1940-1956, Londres \& New York, Leicester University Press.

FAYOLLE Sandra, 2003, «Danielle Casanova et les enjeux de mémoire », in: Mechtild GilzMER, Christine LÉvisSE-TouZÉ \& Stefan MARTEns (dir.), Les femmes dans la Résistance en France. Actes du colloque international de Berlin, 8-10 octobre 2001, Paris, Tallandier.

GadDIS John L., 2005, The Cold War: A New History, New York, Penguin Press.

GINARD David, 2005, Matilde Landa. De la Institución Libre de Enseñanza a las prisiones franquistas. Barcelona, Flor del Viento.

GonZÁlez MArTinez Carmen, 1991, " "Mujeres Antifascistas Españolas", trayectoria histórica de una organización femenina de lucha", in Las mujeres y la Guerra Civil Española, Madrid, Ministerio de Asuntos Sociales, Instituto de la Mujer, Ministerio de Cultura, p. 54-59.

Graham Helen, 1995, "Women and social change », in Helen Graham \& Jo Labany (eds), Spanish Cultural Studies: An Introduction. The Struggle for Modernity. Oxford University Press.

ISER Wolfgang, 1997, « La ficcionalización: dimensión antropológica de las ficciones literarias ", in Antonio GARRIDO (ed.), Teorías de la ficción literaria, Arco Libros, p. $43-68$.

JOLY Maud, 2008, «Las violencias sexuadas en la Guerra Civil española : paradigma para una lectura cultural del conflicto », Historia Social, 61, p. 89-107.

KNIBIEHLER Yvonne, 1997, La révolution maternelle. Femmes, maternité, citoyenneté depuis 1945. Paris, Perrin.

MAgnien Brigitte (ed.), 1995, Hacia una literatura del pueblo: del folletín a la novela, Barcelona, Anthropos.

NADAl Marie-José, 1989, «Femmes de l'exil espagnol. Formation idéologique et action politique des femmes du peuple ayant choisi de s'exiler en France en 1939 », Matériaux pour l'histoire de notre temps, 15, p. 28-37.

NASH Mary, 1981, Mujer y movimiento obrero en España, Barcelona, Fontamara.

—, 1991, «La miliciana: otra opción de combatividad femenina antifascista », in Las mujeres y la Guerra Civil Española, Madrid, Ministerio de Asuntos Sociales, Instituto de la Mujer, Ministerio de Cultura, p. 97-108. 
—, 1999, Rojas. Las mujeres republicanas en la guerra civil, Madrid, Taurus.

NúÑEZ Xosé-Manoel, 2006, ¡Fuera el invasor! Nacionalismos y movilización bélica durante la guerra civil espanola (1936-1939), Madrid, Marcial Pons.

RIPA Yannick, 1997, «Le mythe de Dolores Ibárruri », CLIO. Histoire, Femmes et Sociétés, 5, p. 147-155

Roca Jordi, 1996, De la pureza a la maternidad: la construcción del género femenino en la postguerra española, Madrid, Ministerio de Cultura.

Rousseau Renée, 1983, Les femmes ronges. Chronique des années Vermeersch. Paris, Albin Michel.

TAVERA Susana, 2005, «La memoria de las vencidas: política, género y exilio en la experiencia republicana », Ayer, 60, p. 197-224.

Trat Josette, 2002, "Aux racines de l'idéologie 'familialiste' du PCF », in: Christine Delphy \& Sylvie Chaperon (dir.), Cinquantenaire du Deuxième Sexe. Colloque international Simone de Beauvoir, Paris, Éditions Syllepse, p. 380-386.

YUSTA Mercedes, 2004, «Rebeldía familiar, compromiso invididual, acción colectiva. Las mujeres en la resistencia al franquismo durante los años cuarenta », Historia del Presente, 4, p. 63-93.

—, 2009, "Madres Coraje » contra Franco. La Unión de Mujeres Españolas en el exilio francés (1941-1950), Madrid, Cátedra. 